\title{
XXXVII. The destruction of the fluorescence of iodine and bromine vapour by other gases
}

\section{R.W. Wood}

To cite this article: R.W. Wood (1911) XXXVII. The destruction of the fluorescence of iodine and bromine vapour by other gases, Philosophical Magazine Series 6, 21:123, 309-313, DOI: $10.1080 / 14786440308637036$

To link to this article: http://dx.doi.org/10.1080/14786440308637036

曲 Published online: 21 Apr 2009.

Submit your article to this journal ¿

Џ Article views: 5

Q View related articles $\square$

Citing articles: 3 View citing articles 5 
Destruction of Fluorescence of Iodine and Bromine Vapour. 309

Let $d=d^{\prime}$ when $\mu=1$.

Then evidently $\quad d=\mu d^{\prime}$.

For glass lenses of $10 \mathrm{~cm}$. focus, $d=20 \mathrm{~cm}$., $d^{\prime} \doteqdot 27$, when $\mu=4 / 3$. Therefore it is quite possible to measure both of these lengths to a high degree of accuracy. In the apparatus used, the two lenses are fitted on the ends of two tubes sliding one within the other through a stuffing-box. The apparatus is mounted on the table of a spectrometer whose telescope and collimator have been previously focussed for parallel light. The distance between the lenses is adjusted so that a clear image of the collimator slit is observed in the telescope with the tube $(a)$ full of air, $(b)$ full of water or other liquid. Index marks are placed on the tubes and the distance between these may be measured with vernier calipers, or by means of a travelling microscope if great accuracy is desired.

XXXVII. The Destruction of the Fluorescence of Iodine and Bromine Vapour by other Gases. By R. W. Wood *

[Plate III.]

A $\mathrm{N}$ extended study of the fluorescence of sodium, A potassium, mercury and iodine vapour has shown that the intensity of the emitted light is greatly reduced if air, or some other chemically inert gas, is present. A quantitative study of the phenomenon, showing the relation between the intensity of the fluorescence and the pressure and molecular weight of the foreign gas, is much to be desired as a means of testing any hypothesis which may be made regarding the action of the gas upon the radiating molecules. The vapour of iodine is especially suited to the work, since its fluorescence can be observed at room temperature in glass bulbs, and the conditions of pressure, density, \&c. can be accurately determined, which is nearly or quite impossible with sodium vapour.

A satisfactory theory of the phenomenon should not only explain the destruction of the fluorescence by the inert gas, but also the failure of bromine to show any trace of fluorescence when under the same conditions as iodine vapour. Its absorption spectrum is very similar, and yet it usually remains quite dark even under the most powerful excitation.

Some years ago I suggested the hypothesis that the molecule might be capable of storing up a certain amount of

* Communicated by the Author. 
energy without the emission of light, but that a saturation point must be reached eventually, after which there will be an emission of radiation. If we assume that on collision with another molecule the energy absorbed by the molecule is transformed into heat, the internal energy dropping back to its original value, and the molecular velocity increasing in proportion, it is clear that if the mean free path is traversed before the saturation point is reached, there will be no fluorescence. On this hypothesis we should explain the failure of bromine to fluoresce by ascribing to the bromine molecule a greater capacity for storing energy. In other words, the path cannot be increased sufficiently to allow the saturation point to be reached before a collision occurs. It seemed possible to test this theory by experiment. By sufficiently increasing the length of free path, we ought to be able to observe fluorescence, provided that a sufficient number of molecules remain to produce a visible illumination. A small amount of bromine vapour was introduced into a bulb, and condensed upon the wall by the application of solid carbon dioxide and ether. The bulb was then exhausted to the highest possible degree and sealed. On warming it to room temperature the bromine vaporized, and though it was so highly rarefied that it showed no colour, no fluorescence could be detected.

Sunlight was now concentrated at the centre of the bulb by means of a portrait lens having a ratio of focus to aperture of $2 \cdot 3$. Even in a dark room with careful screening off of diffused light, no fluorescence could be detected. The outside of the bulb was now touched with a piece of. solid carbon dioxide, which gradually condensed the bromine upon the wall. In two or three seconds a faint green fluorescence appeared, which vanished almost immediately, owing to the complete removal of the bromine vapour. There appears then to be one density at which bromine shows a visible fluorescence. At higher densities collisions destroy it, at lower, there are too few molecules present. This appears to be in accord with our hypothesis regarding absorption of energy, saturation point, \&c. ; but more recent work, made in collaboration with J. Franck, has shown that another factor comes into play. The question will be considered again in the paper immediately following the present one.

The method of observing the iodine fluorescence has been so improved that it is now possible to demonstrate it to the largest audience. A large bulb 15 or $20 \mathrm{cms}$. in diameter is prepared by drawing down the neck of a round-bottomed flask, which should be most carefully cleaned with aqua regia 
of Iodine and Bromine Vapour by other Gases. $\quad 311$

and distilled water, a voiding the use of alcohol and ether for drying. A few small crystals of iodine are now introduced into the flask, the neck drawn down to a $1 \mathrm{~mm}$. capillary at one point, and the flask thoroughly exhansted with a Gaede or other mercurial pump. It is most important to have a very perfect vacuum (less than $.01 \mathrm{~mm}$.), and it is usually necessary to keep the pump running for 15 or 20 minutes to secure this, if the capillary is narrow. The flask is now sealed, and can be used for demonstration purposes at any time. It requires no heating, for the iodine fluorescence is brightest at the pressure which the vapour has at room temperature. We have only to hold the bulb in the converging beam furnished by the condensers of a large projecting lantern, or the condensed beam from a heliostat. If a lantern is used it is best to throw the beam upwards, as the reflexions give less trouble. The intensely brilliant cone of yellowish-green fluorescent light can be seen from the back of the largest lecture hall. The aberration of the spherical lenses is well shown as well.

The bulbs used in the photometric work were smaller, having diameters of about $7 \mathrm{~cm}$.

The fluorescence was excited by sunlight reflected into a dark room by a large heliostat, and concentrated to a focus at the centre of the bulb by a Voigtländer portrait objective of $12 \mathrm{~cm}$. aperture and $27 \mathrm{~cm}$. focus (F. 2:3). The work was done only on very clear days when the sky was free from haze and clouds. A Welsbach light was used as a standard, its colour being brought to a match with that of

Fig. 1.

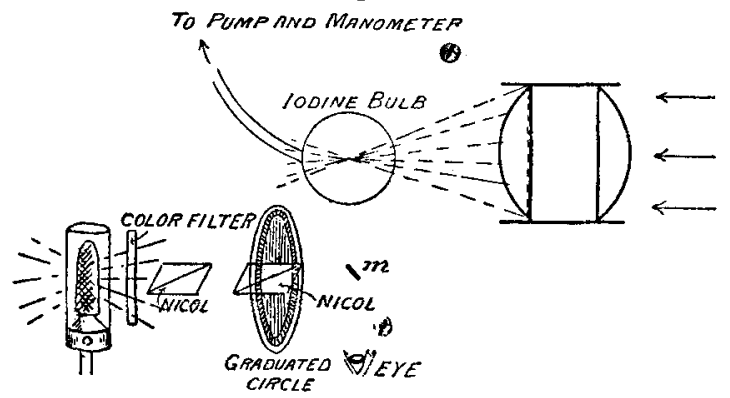

the iodine fluorescence by a combination of pale cobalt glass and a dilute solution of bichromate of potash. The arrangement of the apparatus is shown in fig. 1. Between the eye and the brightest part of the fluorescent cone is mounted a small mirror $m$, measuring about $1 \times 3 \mathrm{~mm}$., made by silvering a 


\section{Prof. R. W. Wood on Destmuction of the Fluorescence}

piece of plate glass and breaking off thin scales by tapping the edge with a hammer, striking the blow in a direction nearly parallel to the silvered surface. The silvered scales obtained in this way usually have one ed ge of razor sharpness, and this edge forms the vanishing line of the photometer. The silvered mirror reflects to the eye the light from the comparison source, which is placed in such a position that the glass sliver cuts across the fluorescent cone at its brightest point. By rotating the graduated nicol prism the intensities may be perfectly matched, the sharp edge of the illuminated mirror vanishing. The intensity of the fluorescence is measured by the square of the cosine of the angle through which the nicol has been turned, measured from the position of complete extinction.

The bulb containing the iodine was in communication with a Gaede pump, a manometer, and a reservoir of the gas under investigation. The bulb was first highly exhausted and the intensity of the fluorescence measured. Air or some other gas was now introduced until the manometer showed a pressure of $1 \mathrm{~mm}$., and the intensity again measured. The pressure of the gas was increased by progressively small steps, the intensity being measured for each pressure. Plotting the results, with the intensities as ordinates and the pressures as abscisse, gives us a curve showing the rate at which the intensity of the fluorescence decreases with increasing gas pressure. Experiments were made with air, hydrogen, carbon dioxide, and ether vapour. Several series were made with each gas, and the results were in good agreement.

The curves are reproduced in Plate III. together with curves obtained with other gases, which will be discussed in the following paper.

An examination of these curves showed that the hypothesis of free path and saturation point would not represent the facts, and that some other factor must be taken into consideration.

The effectiveness of the gas in destroying the fluorescence appeared to increase with its molecular weight, but was by no means proportional to it. For example, the intensity of the fluorescence was reduced from 45 to 85 by $3 \mathrm{~mm}$. of ether vapour, $7 \mathrm{~mm}$. $\mathrm{CO}_{2}, 11.5 \mathrm{~mm}$. air, and $24 \mathrm{~mm}$. of hydrogen. It seems clear from these results that some other property of the gas than its molecular weight must be operative.

In the case of the fluorescence of anthracene, Elston found that the presence of hydrogen and nitrogen was almost with- 
out influence upon the intensity of the emitted light. Oxygen and $\mathrm{CO}_{2}$, on the other hand, reduced its intensity very rapidly with increasing pressure. This I have provisionally ascribed to "incipient chemical action" which of course in reality means nothing at all. It was found that no permanent chemical change took place, for on cooling the bulbs the anthracene condensed upon the walls, and none of the oxygen had disappeared. At high temperatures, however, the oxygen acts upon the anthracene, and it seemed possible that the first stage of the process might occur at the lower temperatures used in the fluorescence experiments, the process reversing as soon as the bulbs were cooled. This is what I called "incipient chemical action." The probable real nature of the action will be given in the subsequent paper.

The only difficulty found in measuring the intensity of the fluorescent light resulted from the slight change of colour which occurred when the intensity was considerably reduced. The colour always became slightly reddish at the higher pressures, though it was only conspicuous when seen in the photometer, the colour match not remaining perfect.

The gases apparently weaken the green portion of tho fluorescent spectrum to a greater extent than the red. This is a very interesting and important matter, which will be discussed in the following paper.

Experiments were also made with a highly exhausted bulb, the vapour pressure of the iodine being varied by immersing a side tabe in freezing mixtures of various temperatures, or heating the entire bulb in a water-bath. The values found below room temperature are given in the following table, and are shown in graphical form on Plate III. (small inset) :-

$\begin{array}{ccc}\text { Temp. } & \text { Intensity. } & \\ 19 & 43 & \text { The relation is very } \\ 6^{\circ} & 29 & \text { nearly linear. } \\ 0^{\circ} & 22 & \\ -10^{\circ} & 11 & \\ -15 & 6 & \\ -20 & 2 & \end{array}$

Above room temperature no perceptible increase could be detected. The intensity remained about the same up to $30^{\circ}$, after which it gradually decreased. At high pressures the fluorescence disappeared entirely

It is probable that increased absorption compensates very nearly for any increase that may occur above room temperature. Accurate measurements were impossible on account of the change of colour due to the absorption of the iodine vapour between the fluorescent cone and the eye.

Phil. May. S. 6. Vol. 21. No. 123. March 1911. Y 
WOOD.

Phil. Mag. Ser. 6. Vol, 21, Pl. III.

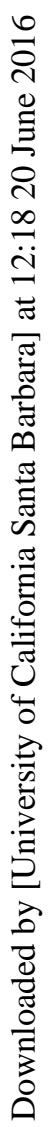

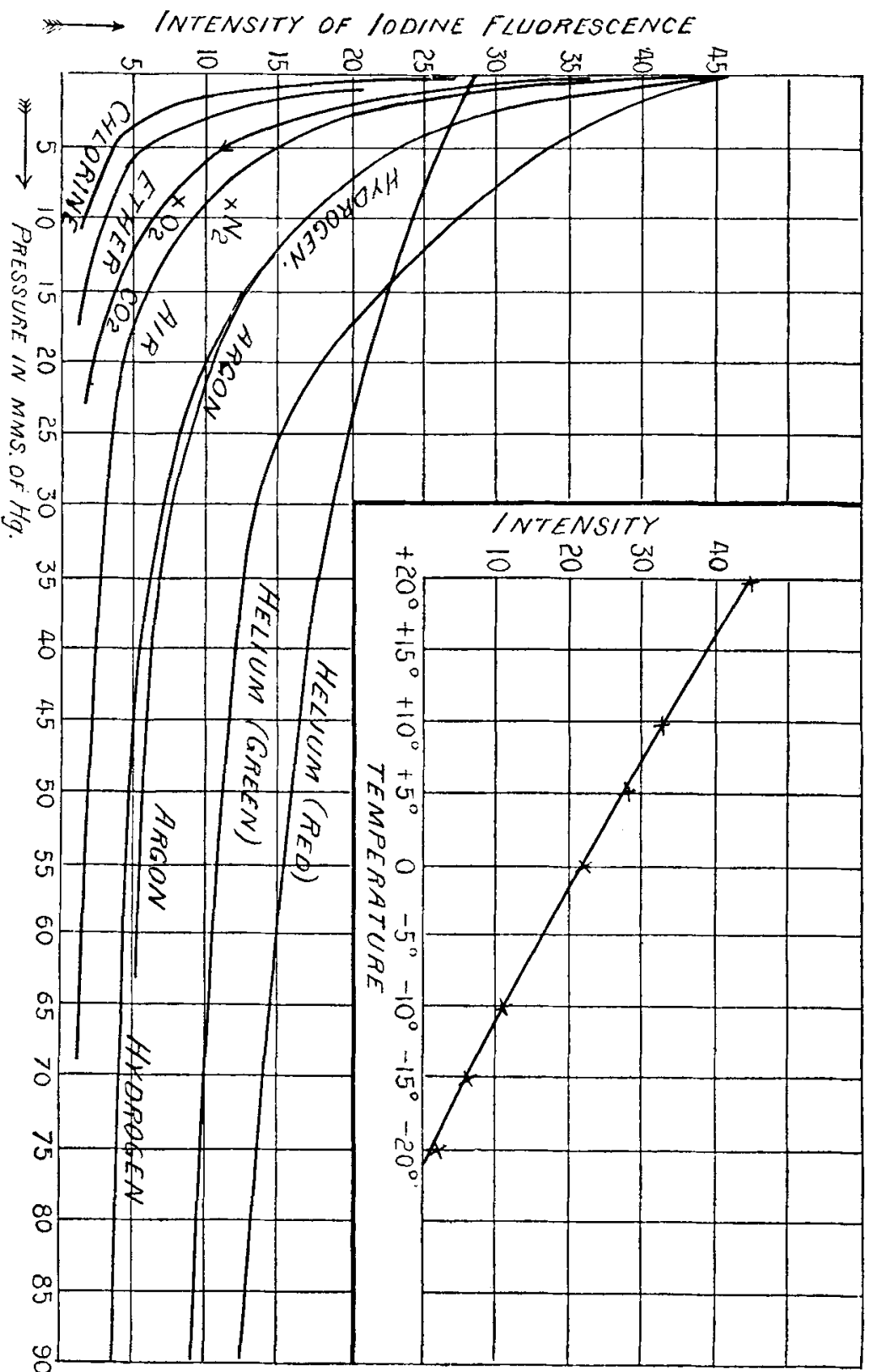

